\title{
Maximum directed cuts in digraphs with degree restriction
}

\author{
Jenő Lehel * $\quad$ Frédéric Maffray ${ }^{\dagger} \quad$ Myriam Preissmann ${ }^{\ddagger}$
}

November 15, 2018

\begin{abstract}
For integers $m, k \geq 1$, we investigate the maximum size of a directed cut in directed graphs in which there are $m$ edges and each vertex has either indegree at most $k$ or outdegree at most $k$.
\end{abstract}

\section{Introduction}

We deal with directed graphs, called here digraphs, without loops and parallel edges. An edge $x y$ of a digraph is interpreted as an arc or an arrow going from the starting vertex or tail $x$ to the end vertex or head $y$. The indegree and the outdegree of a vertex $v \in V(D)$ is respectively defined as $d_{D}^{-}(v)=\mid\{z v \in$ $E(D) \mid z \in V(D)\} \mid$ and $d_{D}^{+}(v)=|\{v w \in E(D) \mid w \in V(D)\}|$.

Let $X, Y$ be a partition of the vertex set $V(D)$ of a digraph $D$. The edge set $\{x y \in E(D) \mid x \in X, y \in Y\}$ is called a directed cut. Clearly a directed cut of a digraph $D$ does not contain a directed path on three vertices $\left(\right.$ a $\left.P_{3}\right)$. On the other hand every directed $P_{3}$-free subgraph of $D$ is the subgraph of some directed cut. Thus when estimating the size of maximum directed cuts we must find directed $P_{3}$-free subgraphs as large as possible. The size of a cut is its cardinality, the size of a digraph is the cardinality of its edge set.

Discussions in [1] show that a digraph $D$ of size $m$ has a cut of size $\frac{1}{4} m+$ $\Theta\left(\mathrm{m}^{1 / 2}\right)$. Furthermore, if the outdegree of each vertex of $D$ is at most $k$, then $D$ has a cut of size at least $\left(\frac{1}{4}+\frac{1}{8 k+4}\right) m$. In [6], lower bounds for the largest directed cuts were asked for a family of digraphs with constrained indegree or outdegree. Let $D(k, \ell)$ be the family of all digraphs in which every vertex has either indegree at most $k$ or outdegree at most $\ell$ (that is $d^{-}(v) \leq k$ or $d^{+}(v) \leq \ell$,

\footnotetext{
*Department of Mathematical Sciences, The University of Memphis, Tennessee, USA and Computer and Automation Research Institute of the Hungarian Academy of Sciences, Budapest, Hungary; jlehel@memphis.edu

${ }^{\dagger}$ CNRS, Laboratoire G-SCOP, 46 avenue Félix Viallet, 38031 Grenoble Cedex, France; frederic.maffray@g-scop.inpg.fr

${ }^{\ddagger}$ CNRS, Laboratoire G-SCOP, 46 avenue Félix Viallet, 38031 Grenoble Cedex, France; myriam.preissmann@g-scop.inpg.fr
} 
for all $v \in V(D)$ ). Note that a directed cut (or any directed $P_{3}$-free graph) forms a graph that belongs to $D(0,0)$.

In Section 2 we consider the case $k=\ell=1$ and discuss the size of the maximum directed cut of digraphs in $D(1,1)$. It was proved in [1] that every acyclic digraph of size $m$ in $D(1,1)$ has a directed cut of at least $2 m / 5$ edges. From a result of Bondy and Locke [4] it is easy to see that the same lower bound holds for maximum directed cuts in triangle-free subcubic digraphs (a graph is subcubic if it has maximum degree at most three). Our main result in Theorem 1 is the extension of this bound for all digraphs in $D(1,1)$ as follows: if $D$ contains at most $t$ pairwise disjoint directed triangles, then $D$ has a directed cut of size at least $(2 m-t) / 5$. The proof yields a polynomial algorithm which actually finds a directed cut of that size (Corollary 2).

Theorem 1 implies that every digraph of size $m$ in $D(1,1)$ has a directed cut with at least $m / 3$ edges (a result first proved in [1]). Furthermore, every connected digraph of size $m$ in $D(1,1)$ has a directed cut with at least $7 m / 20$ edges (see Theorem 5).

In Section 3 we consider digraphs in $D(k, k)$ for any $k$. First we prove a decomposition property in Theorem 8 , the edge set of every digraph in $D\left(p_{1}+\right.$ $\left.p_{2}, p_{1}+p_{2}\right)$ can be partitioned into two subgraphs one in $D\left(p_{1}, p_{1}\right)$ and the other in $D\left(p_{2}, p_{2}\right)$. In Theorem 10 we prove the lower bound $(2 k-1) m /(2 k+1)$ on the maximum size of a subgraph of $D$ belonging to $D(k-1, k-1)$. It is worth noting that the regular tournament on $2 k+1$ vertices has no subgraph in $D(k-1, k-1)$ with more than $(2 k-1 / k) m /(2 k+1)$ edges.

In Section 4 we show that if $D \in D(k, k)$ is acyclic and has $m$ edges, then it contains a directed cut of size at least $\left(\frac{1}{4}+\frac{1}{8 k+4}\right) m$ (see Theorem 12). It is worth noting that in a digraph $D \in D(k, k)$ one cannot guarantee a directed cut of size larger than that proportion. This is shown by the regular tournament on $2 k+1$ vertices, which has no directed cut of size more than $\left(\frac{1}{4}+\frac{1}{8 k+4}\right)\left(\begin{array}{c}2 k+1 \\ 2\end{array}\right)$ (see in [1). For $k=2$ this ratio is $3 m / 10$. In Theorem 13 we can show that actually every digraph $D \in D(2,2)$ with $m$ edges has a directed cut of size at least $3 \mathrm{~m} / 10$. In the proof of Theorems 12 and 13 we use an elementary counting method similar to those applied in [1].

Section 5 concludes with open problems for further consideration. A challenging question whose answer we would like to see the most is whether Theorem 12 remains true for all digraphs in $D(k, k)$, and for every $k \geq 3$.

\section{Maximum directed cut of digraphs in $D(1,1)$}

It was proved in 1 that every acyclic digraph of size $m$ in $D(1,1)$ has a directed cut of at least $2 \mathrm{~m} / 5$ edges. This is not true for all digraphs in $D(1,1)$. For example the directed triangle, which is a member of $D(1,1)$, has no directed cut with two edges. Hence there are digraphs of size $m$ with maximum directed cut not larger than $m / 3$. On the other hand, it was shown in [1] that the edge set of every digraph $D \in D(1,1)$ has a decomposition into three directed cuts (see Theorem 7 below), hence $D$ always contains a directed cut of size $m / 3$. 
One might conclude that the ratio $\mathrm{m} / 3$ cannot be improved to $2 \mathrm{~m} / 5$ in general, and the graph that consists of disjoint directed triangles is an obvious example showing that. Actually the following example shows that, for infinitely many values of $m$, there are even connected digraphs in $D(1,1)$ of size $m$, that contain no cut of size $3 \mathrm{~m} / 8$.

Example 1. For $i=1, \ldots, k$ let $H_{i}$ be a directed path with five vertices $\left(u_{i}, v_{i}, w_{i}, x_{i}, y_{i}\right)$ plus the chord $v_{i} x_{i}$, such that the $H_{i}$ 's are pairwise disjoint. Add $k+1$ directed triangles $\left(y_{i}, u_{i+1}, z_{i}\right)$, for $i=0, \ldots, k$, where $y_{0}, u_{k+1}$ and $z_{0}, z_{1}, \ldots, z_{k}$ are distinct new vertices. The obtained graph $H$ has $m=8 k+3$ edges and its maximum directed cut has size $3 k+1=(3 m-1) / 8$.

In spite of the evidence that the maximum directed cut size to edge count ratio $2 / 5$ cannot be achieved, we show in the next theorem that $1 / 3$ improves to $2 / 5$, in some sense, for all digraphs in $D(1,1)$.

Theorem 1. Let $D$ be a digraph in $D(1,1)$ with $m$ edges, and let $t$ be the maximum number of pairwise disjoint directed triangles in $D$. Then $D$ has a directed cut of size at least $(2 m-t) / 5$.

Proof. The claim is clearly true for $m \leq 3$ and $t=0$. If $m=3$ and $t=1$, then $D$ is the directed triangle and any edge of the triangle forms a directed cut of size $1=(2 m-t) / 5$. Now let $D$ be a counterexample with $m \geq 4$ edges, and assume that the theorem is true for all digraphs in $D(1,1)$ with at most $m-1$ edges. Clearly $D$ is connected.

Let $D^{+}$be the subgraph of $D$ induced by $V^{+}=\left\{v \in V(D) \mid d^{+}(v) \geq 2\right\}$; and let $D^{-}$be the subgraph of $D$ induced by $V^{-}=\left\{v \in V(D) \mid d^{-}(v) \geq 2\right\}$. Notice that $v \in V^{+}$implies that $d^{-}(v) \leq 1$ and $v \in V^{-}$implies $d^{+}(v) \leq 1$.

Because $D \in D(1,1)$, if two directed triangles of $D$ have a common vertex, then they must share a common edge. Moreover, if a triangle intersects with at least two other triangles, then they all share the same common edge. The following property of triangles will be useful.

Claim 1.1. Every directed triangle of $D$ is contained in $D^{+}$or in $D^{-}$.

Assume that $T=(x, y, z)$ is a directed triangle with $d^{-}(x)=1$ and $d^{+}(y)=$ 1. Remove the edges of $T$ from $D$. The graph $D^{\prime}$ that remains has $m^{\prime}=m-3$ edges, and the maximum number $t^{\prime}$ of disjoint triangles in $D^{\prime}$ satisfies $t^{\prime} \leq t-1$. By induction, $D^{\prime}$ contains a directed cut $K$ of size at least $\left(2 m^{\prime}-t^{\prime}\right) / 5 \geq$ $(2 m-t) / 5-1$. Obviously, $K \cup\{x y\}$ is still a directed $P_{3}$-free subgraph of $D$ containing $(2 m-t) / 5$ edges, a contradiction. Therefore, either $d^{+}(w) \geq 2$ for all $w \in\{x, y, z\}$ or $d^{-}(w) \geq 2$ for all $w \in\{x, y, z\}$. In the first case $T \subset D^{+}$ and in the second case $T \subset D^{-}$. Thus Claim 1.1 holds.

Let $A, B \subset E(D)$ be a pair of disjoint edge sets such that every directed $P_{3}$ in $D$ that has one edge in $A$ has its second edge in $B$. Note that this implies that $A$ contains no directed $P_{3}$. We call any such pair $A, B \subset E(D)$ a reducing 
pair. It is clear that if $K$ is any directed cut in the digraph $D \backslash(A \cup B)$, then $K \cup A$ is a directed $P_{3}$-free subgraph of $D$. The following claim will be used several times in the induction step.

Claim 1.2. $D$ has no reducing pair $A, B \subset E(D)$ with $|B| \leq \frac{3}{2}|A|$.

Suppose that $A, B \subset E(D)$ is a reducing pair with $|B| \leq \frac{3}{2}|A|$. Let $K$ be a largest directed cut in the digraph $D^{\prime}=D \backslash(A \cup B)$. Then $K \cup A$ is a directed $P_{3}$-free subgraph of $D$. Digraph $D^{\prime}$ has $m^{\prime}=m-|A \cup B|$ edges, hence it follows by induction that $|K| \geq\left(2 m^{\prime}-t\right) / 5$. We obtain

$$
|K \cup A| \geq \frac{2 m^{\prime}-t}{5}+|A| \geq \frac{2 m-t}{5}-\frac{2}{5}(|A|+|B|)+|A| \geq \frac{2 m-t}{5},
$$

which contradicts the assumption that $D$ is a counterexample to the theorem. Thus Claim 1.2 holds.

Claim 1.3. Each of $D^{+}$and $D^{-}$is a disjoint union of directed cycles. Furthermore, every vertex in $D^{+}$or $D^{-}$is incident with exactly one edge of $D \backslash$ $\left(D^{+} \cup D^{-}\right)$.

Let $C$ be any connected component of $D^{-}$. We show that $C$ is a directed cycle. By the definition of $D^{-}, C$ is either a rooted tree with all edges directed towards the root, or a function graph which is a rooted tree plus an edge from the root to some vertex of the tree.

If $C$ is not a directed cycle, then it is either a singleton vertex $v_{0}$ or it has a leaf $v_{0}$. In each case, because $v_{0}$ is in $V^{-}$, there exist distinct edges $e_{1}=v_{1} v_{0}, e_{2}=v_{2} v_{0}$ of $D$. Furthermore, $d^{+}\left(v_{0}\right) \leq 1$, thus at most one edge $f_{0}$ leaves $v_{0}$. Since $v_{1}, v_{2}$ are not in $C$, they are not in $V^{-}$, hence at most one edge enters each, say $f_{1}$ and $f_{2}$, respectively. Note that both edges exist in $A=\left\{e_{1}, e_{2}\right\}$, but any edge from the set $B=\left\{f_{0}, f_{1}, f_{2}\right\}$ might actually not exist. In either case, $A, B$ form a reducing pair with $|B| \leq \frac{3}{2}|A|$, contradicting Claim 1.2. Thus every component of $D^{-}$is a directed cycle. Furthermore, if there are two edges $e_{1}, e_{2}$ of $D \backslash\left(D^{+} \cup D^{-}\right)$at some vertex $v_{0} \in V^{-}$, then one obtains a contradiction using the same reducing pair.

An analogous argument shows that every connected component $C$ of $D^{+}$is a directed cycle with exactly one edge of $D \backslash\left(D^{+} \cup D^{-}\right)$at each vertex of $C$. Thus Claim 1.3 holds.

Note that, due to Claims 1.1 and 1.3, all directed triangles of $D$ are among the cycles of $D^{+}$and $D^{-}$.

Claim 1.4. All directed cycles in $D^{+}$and $D^{-}$have odd length.

Suppose the contrary, and let $C=\left(x_{1}, x_{2}, \ldots, x_{2 p}\right)$ be a directed cycle, say in $D^{+}$. For every $i=1, \ldots, p$ let $e_{2 i-1}, e_{2 i}$ be the two edges going out from $x_{2 i}$, such that $e_{2 i}=x_{2 i} x_{2 i+1}$, and call $y_{i}$ the end vertex of $e_{2 i-1}$. Then $y_{i} \in V \backslash V^{+}$, therefore there is at most one edge $g_{i}$ going out from $y_{i}$. For every $i=1, \ldots, p$, let $f_{2 i-1}, f_{2 i}$ be the two edges going out from $x_{2 i-1}$, such that $f_{2 i-1}=x_{2 i-1} x_{2 i}$. 
Let $A=\left\{e_{1}, \ldots, e_{2 p}\right\}$ and $B=\left\{f_{1}, \ldots, f_{2 p}\right\} \cup\left\{g_{1}, \ldots, g_{p}\right\}$. Observe that $A, B$ are disjoint and that $B$ contains one edge of each directed $P_{3}$ of $D$ that has an edge in $A$. Therefore $A, B$ is a reducing pair, with $|B| \leq \frac{3}{2}|A|$, contradicting Claim 1.2 Thus Claim 1.4 holds.

Claim 1.5. $D^{+}$and $D^{-}$have the same number of vertices, say this number is $k$, and $D \backslash\left(D^{+} \cup D^{-}\right)$is the union of $k$ disjoint edges going from $D^{+}$to $D^{-}$.

We shall prove that $V^{0}=V(D) \backslash\left(V^{-} \cup V^{+}\right)=\emptyset$. Assume on the contrary that $V^{0} \neq \emptyset$. By the connectivity of $D$, there is a vertex $y \in V^{0}$ adjacent to some vertex of $D^{+} \cup D^{-}$. By symmetry, we may assume that $y z$ is an edge for some $z \in V^{-}$. Let $C \subseteq D^{-}$be the directed cycle containing $z$, let $C$ have length $2 \ell+1$, with $\ell \geq 1$. We call $(\ell+1)$-set any subset $L \subset V(C)$ such that $V(C) \backslash L$ is a maximum independent set of $C$. Note that for any two vertices $x, y$ of $C$ there exists an $(\ell+1)$-set that contains both $x, y$.

If $d^{-}(y) \neq 0$, then let $e_{0}=x y$, and let $g_{0}$ be an edge going into $x$ if it exists. (Note that $y \notin V^{-}$implies $x \notin V^{-}$.) Let $L \subset V(C)$ be an $(\ell+1)$-set of $C$ not containing $z$ and define:

$$
\begin{aligned}
B_{1} & =\left\{f \in E(C) \mid f=w w^{\prime} \text { for some } w \in L\right\}, \\
A & =\left\{e_{0}\right\} \cup\left(E(C) \backslash B_{1}\right) \cup\{e \notin E(C) \mid e=v w \text { for some } w \in L\}, \\
B_{2} & =\left\{g \in\left(E(D) \backslash B_{1}\right) \mid g=u w \text { such that } w v \in A\right\} .
\end{aligned}
$$

Observe that $A$ contains no directed $P_{3}$ and that every directed $P_{3}$ with one edge in $A$ has its other edge in $B=B_{1} \cup B_{2}$. So $A, B$ is a reducing pair. Since $|A|=2 \ell+2,\left|B_{1}\right|=\ell+1$, and $\left|B_{2}\right| \leq 2 \ell+2$, we have $|B| \leq \frac{3}{2}|A|$, contradicting Claim 1.2 . define:

If $d^{-}(y)=0$, then let $L \subset V(C)$ be an $(\ell+1)$-set of $C$ containing $z$, and

$$
\begin{aligned}
B_{1} & =\left\{f \in E(C) \mid f=w w^{\prime} \text { for some } w \in L\right\}, \\
A & =\left(E(C) \backslash B_{1}\right) \cup\{e \notin E(C) \mid e=v w \text { for some } w \in L\}, \\
B_{2} & =\left\{g \in\left(E(D) \backslash B_{1}\right) \mid g=u w \text { such that } w v \in A\right\} .
\end{aligned}
$$

Again, $A$ and $B=B_{1} \cup B_{2}$ form a reducing pair. We have $|A|=2 \ell+1,\left|B_{1}\right|=$ $\ell+1$ and $\left|B_{2}\right|=2 \ell$ since no edge enters into $y$. Hence $|B|<\frac{3}{2}|A|$, contradicting Claim 1.2 Then Claim 1.5] follows from the second part of Claim 1.3 .

Call $M$ the (loopless) bipartite multigraph obtained by contracting of every directed cycle into one vertex.

Claim 1.6. $M$ is a simple graph.

Suppose on the contrary that there are at least two edges from the cycle $C^{+} \subseteq D^{+}$to the cycle $C^{-} \subseteq D^{-}$. By Claim 1.5. $C^{+}$is an odd cycle, thus there exist edges $u x, v y \in E(D)$ with $u, v \in V\left(C^{+}\right), x, y \in V\left(C^{-}\right)$such that $\left(u, b_{1}, \ldots, b_{2 q}, v\right)$ is a directed subpath of $C^{+}$, and no vertex $b_{i}$ has an edge to $C^{-}\left(q=0\right.$ means that $u v$ is an edge of $\left.C^{+}\right)$. 
Let $C^{-}$have length $2 \ell+1$ (it is an odd cycle by Claim 1.5). Obviously there exists an $(\ell+1)$-set $L \subset V\left(C^{-}\right)$including $x$ and excluding $y$. Define:

$$
\begin{aligned}
& B_{1}=\left\{f \in E\left(C^{-}\right) \mid f=w z \text { for some } w \in L\right\}, \\
& A_{0}=\left(E\left(C^{-}\right) \backslash B_{1}\right) \cup\left\{e \notin E\left(C^{-}\right) \mid e=z w \text { for some } w \in L\right\}, \\
& B_{2}=\left\{g \in\left(E(D) \backslash B_{1}\right) \mid g=w z \text { such that } z w^{\prime} \in A_{0}\right\} .
\end{aligned}
$$

Let $e_{0}^{\prime}=u b_{1}, f_{0}^{\prime}=v w$ where $w \in V\left(C^{+}\right), g_{0}^{\prime}=v y$, and define:

$$
\begin{aligned}
& A_{0}^{\prime}=\left\{e_{0}^{\prime}\right\} \cup\left\{e^{\prime} \in E(D) \mid e^{\prime}=b_{2 i} z, 1 \leq i \leq q\right\}, \\
& B_{1}^{\prime}=\left\{f_{0}^{\prime}\right\} \cup\left\{f^{\prime} \in E(D) \mid f^{\prime}=b_{2 i-1} z, 1 \leq i \leq q\right\}, \\
& B_{2}^{\prime}=\left\{g^{\prime} \in\left(E(D) \backslash B_{1}^{\prime}\right) \mid g^{\prime}=z w^{\prime} \text { such that } b z \in A_{0}^{\prime}\right\} \backslash\left\{g_{0}^{\prime}\right\} .
\end{aligned}
$$

Observe that the set $A=A_{0} \cup A_{0}^{\prime}$ contains no directed $P_{3}$, and every directed $P_{3}$ with an edge in $A$ has its other edge in $B=B_{1} \cup B_{2} \cup B_{1}^{\prime} \cup B_{2}^{\prime}$ from $D$. Hence $A, B$ form a reducing pair. We have $\left|A_{0}\right|=2 \ell+1,\left|B_{1}\right|=\ell+1$, $\left|B_{2}\right|=2 \ell+1,\left|A_{0}^{\prime}\right|=2 q+1,\left|B_{1}^{\prime}\right|=2 q+1,\left|B_{2}^{\prime}\right|=q$, so $|A|=2(\ell+q+1)$ and $|B|=3(\ell+q+1)=\frac{3}{2}|A|$, contradicting Claim 1.2. Thus Claim 1.6 holds.

Because every vertex of the contraction graph $M$ has degree at least three, $M$ has a cycle. To conclude the proof of the theorem we show that this leads to a contradiction.

Consider a shortest cycle $\gamma \subset M$, and let $\gamma=\left(C_{1}^{+}, C_{1}^{-}, C_{2}^{+}, C_{2}^{-}, \ldots, C_{p}^{+}, C_{p}^{-}\right)$, where, for each $i \in\{1, \ldots, p\}, C_{i}^{+} \subseteq D^{+}$and $C_{i}^{-} \subseteq D^{-}$are cycles of $D$ of odd length. The edges of $\gamma$ correspond to a matching of $D$ from the set $\cup_{i=1}^{q}\left\{u^{i}, v^{i}\right\}$, to the set $\cup_{i=1}^{q}\left\{x^{i}, y^{i}\right\}$, where $u^{i}, v^{i} \in C_{i}^{+}$and $x^{i}, y^{i} \in C_{i}^{-}$. Furthermore, by Claim [1.6 and since $\gamma$ has no chords in $M$, no more edges of $D$ are induced between these cycles. We may assume, so we do, that $\left(u^{i}, b_{1}^{i}, \ldots, b_{2 q_{i}-1}^{i}, v^{i}\right)$, where $q_{i} \geq 1$, is a directed subpath of $C_{i}^{+}$.

Let $2 \ell_{i}+1$ be the length of $C_{i}^{-}$. For every $i=1, \ldots, p$ select an $\left(\ell_{i}+1\right)$-set $L_{i} \subset V\left(C_{i}^{-}\right)$of $C^{-}$such that $x^{i}, y^{i} \in L_{i}$, and define the following sets:

$$
\begin{aligned}
& B_{i}^{1}=\left\{f \in E\left(C_{i}^{-}\right) \mid f=w z \text { for some } w \in L_{i}\right\}, \\
& A_{i}^{1}=\left(E\left(C_{i}^{-}\right) \backslash B_{i}^{1}\right) \cup\left\{e \notin E\left(C_{i}^{-}\right) \mid e=z w \text { for some } w \in L_{i}\right\}, \\
& B_{i}^{2}=\left\{g \in\left(E(D) \backslash B_{i}^{1}\right) \mid g=u z \text { such that } z w \in A_{i}^{1}\right\} .
\end{aligned}
$$

Let $A^{1}=\cup_{i=1}^{p} A_{i}^{1}$ and $B^{1}=\cup_{i=1}^{p}\left(B_{i}^{1} \cup B_{i}^{2}\right)$. We have $\left|A^{1}\right|=\sum_{i=1}^{p}\left|A_{i}^{1}\right|=$ $\sum_{i=1}^{p}\left(2 \ell_{i}+1\right)$, and because $\left|B_{i}^{1}\right|=\ell_{i}+1,\left|B_{i}^{2}\right|=2 \ell_{i}+1$, we obtain $\left|B^{1}\right|=$ $\sum_{i=1}^{p}\left(3 \ell_{i}+2\right)$.

For every $i=1, \ldots, p$, let $e_{i}=u^{i} b_{1}^{i}, f_{i}=b_{2 q_{i}-1}^{i} v^{i}$, and define sets:

$$
\begin{aligned}
& A_{i}^{2}=\left\{e_{i}\right\} \cup\left\{e \in\left(E(D) \mid e=b_{2 j} w, 1 \leq j \leq q_{i}-1\right\},\right. \\
& B_{i}^{3}=\left\{f \in E(D) \mid f=b_{2 j-1} w, 1 \leq j \leq q_{i}\right\} \backslash\left\{f_{i}\right\}, \\
& B_{i}^{4}=\left\{g \in\left(E(D) \backslash B_{i}^{3}\right) \mid g=w z \text { such that } b w \in A_{i}^{2}\right\} \backslash\left\{f_{i}\right\} .
\end{aligned}
$$


Let $A^{2}=\cup_{i=1}^{p} A_{i}^{2}$ and $B^{3}=\cup_{i=1}^{p}\left(B_{i}^{3} \cup B_{i}^{4}\right)$. We have $\left|A^{2}\right|=\sum_{i=1}^{p}\left|A_{i}^{2}\right|=$ $\sum_{i=1}^{p}\left(2 q_{i}-1\right)$, and because $\left|B_{i}^{3}\right|=2 q_{i}-1$ and $\left|B_{i}^{4}\right|=q_{i}-1$, we obtain $\left|B^{3}\right|=$ $\sum_{i=1}^{p}\left(3 q_{i}-2\right)$. Observe that the sets $A=A^{1} \cup A^{2}$ and $B=B^{1} \cup B^{3}$ form a reducing pair. Furthermore, $|A|=\sum_{i=1}^{p}\left(2 \ell_{i}+1+2 q_{i}-1\right)=2 \sum_{i=1}^{p}\left(\ell_{i}+q_{i}\right)$ and $|B|=\sum_{i=1}^{p}\left(3 \ell_{i}+2+3 q_{i}-2\right)=3 \sum_{i=1}^{p}\left(\ell_{i}+q_{i}\right)=\frac{3}{2}|A|$, contradicting Claim 1.2 This concludes the proof of the theorem.

The proof of the theorem can be formulated as an algorithm which, given any digraph $D \in D(1,1)$ with $m$ edges and at most $t$ disjoint directed triangles, constructs a directed cut $K$ of size at least $(2 m-t) / 5$. We sketch such an algorithm here. Start from $K:=\emptyset$. Then apply the following general step. Find the subgraphs $D^{+}$and $D^{-}$. If there is a directed triangle that is not included in $D^{+}$or $D^{-}$, with the notation of Claim 1.1, then set $K:=K \cup\{x y\}$ and iterate with the subgraph $D \backslash\{x y, y z, z x\}$. (When iterating, the subgraphs $D^{+}, D^{-}$ must be updated.) If there is no such directed triangle, then either $D$ violates one of Claims 1.3 1.6, or $D$ satisfies the conditions described after the proof of Claim 1.6, and in either case, the proof of the theorem shows how to find a reducing pair $(A, B)$. Then set $K:=K \cup A$ and iterate the general step with the subgraph $D \backslash(A \cup B)$. The algorithm terminates when $D$ becomes edgeless. Then at termination $K$ is a directed cut of size at least $(2 m-t) / 5$. It is easy to see that all the operations (updating $D^{+}$and $D^{-}$, finding a directed triangle, checking whether $D$ violates one of the claims, determining the structure described after the proof of Claim [1.6) can be done in polynomial time, and there are at most $m$ iterations. Thus we obtain:

Corollary 2. There is a polynomial time algorithm which, given any digraph $D \in D(1,1)$ with $m$ edges and at most $t$ disjoint directed triangles, finds a directed cut in $D$ of size at least $(2 m-t) / 5$.

Corollary 3. If $D \in D(1,1)$ has $m$ edges, then it contains a directed cut of size at least $m / 3$. Moreover $D$ has no directed cut of size larger than $m / 3$ if and only if $D$ is the union of disjoint directed triangles.

Proof. The number of pairwise disjoint directed triangles satisfies $t \leq m / 3$, with equality if and only if $D$ is a union of disjoint directed triangles. Now the claim follows by Theorem 1 , because $(2 m-t) / 5 \geq(2 m-m / 3) / 5=m / 3$.

Corollary 4. If $D \in D(1,1)$ has $m$ edges and no directed triangle, then it contains a directed cut of size at least $2 \mathrm{~m} / 5$.

Results by Bondy and Locke [4] on the bipartite density of (undirected) subcubic graphs are reminescent of our investigations concerning $D(1,1)$. They proved in [4] that a triangle-free subcubic graph has a bipartite subgraph of size at least $4 \mathrm{~m} / 5$. Observe that any triangle-free digraph of maximum degree at most three belongs to $D(1,1)$, and it is obtained from a subcubic graph by orienting its edges. Hence their result implies that such a $D$ has a directed cut of size at least $2 \mathrm{~m} / 5$, the half of $4 \mathrm{~m} / 5$. Corollary 4 shows that this bound 
is valid for the much larger class of digraphs in $D(1,1)$ containing no directed triangle.

Now we show that the lower bound $m / 3$ in Corollary 3 can be surpassed for connected digraphs of $D(1,1)$.

Theorem 5. If $D \in D(1,1)$ is a connected digraph with $m$ edges, and $D$ is not a triangle, then it contains a directed cut of size at least $7 \mathrm{~m} / 20$.

Proof. The proof works by induction on $m$. Let $t$ be the maximum number of pairwise disjoint directed triangles of $D$. By the hypothesis, we have $t=0$ if $m \leq 3$ and $t \leq 1$ if $m=4,5,6$. Thus by Theorem 1 , there is a cut of size at least $1,1,2,2,2,3$, respectively, for $m=1, \ldots, 6$, which matches the corresponding value of $\lceil 7 m / 20\rceil$. Now let $m \geq 7$, and assume that the claim is true for connected graphs with strictly less than $m$ edges. Observe that any $t$ disjoint directed triangles of $D$ have a total of $3 t$ edges, furthermore, by the connectivity of $D$, there are at least $t-1$ more edges between these triangles. Hence we have $m \geq 4 t-1$.

If $m>4 t-1$, or equivalently, if $t \leq m / 4$, then by Theorem [1, $D$ has a cut of size at least $(2 m-t) / 5 \geq(2 m-m / 4) / 5=7 m / 20$ edges as stated.

Assume now that $m=4 t-1$. So $D$ consists of $t$ disjoint directed triangles connected by $t-1$ edges in a tree-like manner. Since we cannot have $t=1$ and $m=3$, we have $t \geq 2$. So there is a directed triangle $T=(x, y, z)$ that is adjacent to exactly one edge, say $x x^{\prime}$, which is adjacent to another directed triangle $T^{\prime}=\left(x^{\prime}, y^{\prime}, z^{\prime}\right)$. (The symmetric argument applies if the orientation of the edge between $T$ and $T^{\prime}$ is $x^{\prime} x$.) Removing from $D$ the vertices and edges of $T$ together with the two edges $x x^{\prime}, x^{\prime} y^{\prime}$, we obtain a connected digraph $D^{\prime}$ with $m^{\prime}=m-5 \geq 2$ edges. By the induction hypothesis, $D^{\prime}$ has a cut $H^{\prime}$ of size at least $7 m^{\prime} / 20=(7 m-35) / 20>7 m / 20-2$ edges. Clearly $H^{\prime} \cup\left\{x x^{\prime}, y z\right\}$ has no directed $P_{3}$, which yields a cut of size at least $7 m / 20$ in $D$.

Just like with Theorem 1, the proof of Theorem [5 can be formulated easily as a polynomial time algorithm (we omit the details). So we have:

Corollary 6. There is a polynomial time algorithm which, given any digraph $D \in D(1,1)$ with $m$ edges, such that no component of $D$ is a directed triangle, finds a directed cut in $D$ of size at least $7 \mathrm{~m} / 20$.

\section{Decompositions of $D(k, k)$}

The problem of covering the edges of a digraph with cuts was proposed in [1. Upper bounds were given for digraphs in $D(k, \ell)$, and the only exact value was determined for $k=\ell=1$.

Theorem 7 ([1]). The edge set of any digraph $D \in D(1,1)$ can be decomposed into at most three cuts. 
Theorem 8. For integers $p_{1}, p_{2} \geq 0$, the edge set of every digraph $D \in D\left(p_{1}+\right.$ $\left.p_{2}, p_{1}+p_{2}\right)$ can be decomposed into two subgraphs $D_{1} \in D\left(p_{1}, p_{1}\right)$ and $D_{2} \in$ $D\left(p_{2}, p_{2}\right)$.

Proof. Since $D$ is in $D\left(p_{1}+p_{2}, p_{1}+p_{2}\right)$, its vertex set $V(D)$ can be partitioned into two sets $X, Y$ such that every vertex $x \in X$ satisfies $d^{-}(x) \leq p_{1}+p_{2}$ and every vertex $y \in Y$ satisfies $d^{+}(y) \leq p_{1}+p_{2}$. Consider the set of edges $B=\{y x \in E(D) \mid y \in Y, x \in X\}$. By the definition of $X$ and $Y$, in the bipartite graph $(X, Y ; B)$ every vertex has degree at most $p_{1}+p_{2}$. By a classical corollary of the König-Hall theorem (see e.g., [7, Prop. 5.3.1]), the edges of $B$ can be colored with $p_{1}+p_{2}$ colors so that any two adjacent edges have different colors. Let $B_{1}$ be the set of edges of $B$ with the first $p_{1}$ colors and $B_{2}$ be the set of edges of $B$ with the remaining colors.

For every vertex $x \in X$, the set $E^{-}(x)$ of edges with end $x$ has size at most $p_{1}+p_{2}$, so it can be partitioned into two sets $E_{1}(x)$ and $E_{2}(x)$ such that, for $j=1,2,\left|E_{j}(x)\right| \leq p_{j}$ and $E^{-}(x) \cap B_{j} \subseteq E_{j}(x)$. Likewise, for every vertex $y \in Y$, the set $E^{+}(y)$ of edges with origin $y$ has size at most $p_{1}+p_{2}$, so it can be partitioned into two sets $E_{1}(y)$ and $E_{2}(y)$ such that, for $j=1,2,\left|E_{j}(y)\right| \leq p_{j}$ and $E^{+}(y) \cap B_{j} \subseteq E_{j}(y)$.

Finally let the set $\{x y \in E(D) \mid x \in X, y \in Y\}$ be partitioned arbitrarily into two sets $F_{1}, F_{2}$. Now, for $j=1,2$, let $D_{j}$ be the subgraph of $D$ whose edge set is $B_{j} \cup F_{j} \cup \bigcup_{x \in V} E_{j}(x)$. The definition of these sets implies that each edge of $D$ lies in exactly one of $D_{1}, D_{2}$ and that $D_{j} \in D\left(p_{j}, p_{j}\right)$ for $j=1,2$. More precisely, for $j=1,2$, in $D_{j}$ every vertex $x \in X$ satisfies $d^{-}(x) \leq p_{j}$ and every vertex $y \in Y$ satisfies $d^{+}(y) \leq p_{j}$.

Corollary 9. The edges of every digraph $D \in D(2,2)$ can be decomposed into two subgraphs $D_{1}, D_{2} \in D(1,1)$.

From Corollary 9 and Theorem 7 it follows that every digraph $D \in D(2,2)$ can be covered with six directed cuts. If there was a decomposition of $D$ into a cut and a digraph in $D(1,1)$, then $D$ would have a cut cover only with four cuts, by Theorem 7 again. Our next example shows that such a decomposition is not always possible.

Example 2. Take two disjoint copies of a regular tournament on five vertices, $G_{1}, G_{2}$, and include all 25 edges directed from $G_{1}$ to $G_{2}$. Thus we obtain a digraph $H \in D(2,2)$. Assume that $K \subset E(H)$ is a cut such that $H^{\prime}=H \backslash K$ is in $D(1,1)$. The regular tournament has no cut with more than three edges, hence $G_{1}$ has a vertex $v_{0}$ such that every edge going into $v_{0}$ is in $E(H) \backslash K$ and at least one edge going out of $v_{0}$ is in $E(H) \backslash K$. Thus $d_{H^{\prime}}^{-}\left(v_{0}\right)=2$, which implies that $v_{0} z \in K$ for all $z \in V\left(G_{2}\right)$ in order to obtain $d_{H^{\prime}}^{+}\left(v_{0}\right) \leq 1$. Then it follows that no edge of $G_{2}$ belongs to $K$, thus $d_{H^{\prime}}^{+}(z)=2$ and $d_{H^{\prime}}^{-}(z) \geq 2$ for all $z \in V\left(G_{2}\right)$, a contradiction.

How large a subgraph belonging to $D(1,1)$ can be found in a digraph $D \in$ $D(2,2)$ ? Corollary 9 implies that $D$ with $m$ edges contains a subgraph in $D(1,1)$ 
with at least $m / 2$ edges. A larger bound will follow from our more general result.

Theorem 10. Every digraph $D \in D(k, k)$ with $m$ edges has a subgraph belonging to $D(k-1, k-1)$ with at least $(2 k-1) m /(2 k+1)$ edges.

Proof. Let $W=\left\{v \in V(D) \mid d_{D}^{-}(v) \leq k\right\}$ and $B=\left\{v \in V(D) \mid d_{D}^{+}(v) \leq k\right\}$. Because $D \in D(k, k)$, we have $V(D)=W \cup B$. We say that $v \in W$ is white, and $v \in B$ is black; note that a vertex may have both colors. An edge $x y \in E(D)$ is called a black tail arrow if $x \in B$, and it is called a white head arrow if $y \in W$. Note that an edge can be both a black tail and a white head arrow. Observe the symmetry of the colors with respect to reversing all arrows in $D$. Due to this symmetry, if a property is verified for white vertices, then the analogous property is true for black vertices with directions reversed.

Let $R \subset E(D)$ be a set of edges such that (a) the graph $D^{\prime}=D \backslash R$ is in $D(k-1, k-1)$, (b) $R$ is minimum among all sets with property (a), and (c) $R$ has the maximum number of black tail arrows and white head arrows (each arrow counted once) among all sets that satisfy (a) and (b). Clearly such a set $R$ exists.

For each edge $e=x y \in R$, we define a critical vertex of $e$ as follows:

$x$ is a critical vertex for $e=x y \in R$ if $d_{D^{\prime}}^{+}(x)=k-1$ and $d_{D^{\prime}}^{-}(x) \geq k$;

$y$ is a critical vertex for $e=x y \in R$ if $d_{D^{\prime}}^{-}(y)=k-1$ and $d_{D^{\prime}}^{+}(y) \geq k$.

The minimality of $R$ means that at least one of $x, y$ is critical for each edge $e=x y$ of $R$. Note that both $x, y$ may be critical for $e$. For each $e \in R$, let $\operatorname{Crit}(e) \subseteq\{x, y\}$ be the set of critical vertices of $e$. For any subset $X \subseteq R$, define $\operatorname{Crit}(X)=\cup_{e \in X} \operatorname{Crit}(e)$. From here on, the word critical vertex refers to elements of $\operatorname{Crit}(R)$. All critical vertices are in the set $\left\{v \in V(D) \mid d_{D}^{-}(v), d_{D}^{+}(v) \geq\right.$ $k\}$, however not every vertex in that set is critical for some edge of $R$. The main point of the proof is to establish that:

Claim 10.1. $|\operatorname{Crit}(R)| \geq|R|$.

Assume we already know that $|\operatorname{Crit}(R)| \geq|R|$. Then the definition of critical vertices implies that, for every $v \in \operatorname{Crit}(R)$, there are at least $2 k-1$ edges not in $R$ and incident with $v$. Thus for the size of $D$ we have the bound $m \geq|R|+(2 k-1)|R| / 2$, hence $|R| \leq 2 m /(2 k+1)$. So $D^{\prime}$ has at least $m-|R| \geq$ $(2 k-1) m /(2 k+1)$ edges, and the theorem follows. Therefore the rest of the proof consists in proving Claim 10.1.

The definition of critical vertices implies easily the following two claims, whose proof is omitted.

Claim 10.2. If $x \in B$ and $d_{R}^{+}(x) \geq 2$, then $x \notin C r i t(R)$. By symmetry, If $x \in W$ and $d_{R}^{-}(x) \geq 2$, then $x \notin \operatorname{Crit}(R)$.

Claim 10.3. If $e=x y \in R, y \in B$, and $y z \in R$, then Crit $(e)=\{x\}$. By symmetry, if $e=x y \in R, x \in W$, and $u x \in R$, then Crit $(e)=\{y\}$. 
Now we examine the subgraph formed by $R$. Let $A \subseteq R$ be any connected component of $R$ (we use $A$ and $R$ to denote the digraphs defined by the edges in $A$ and $R$, respectively). Note that $\operatorname{Crit}(R)=\operatorname{Crit}(A) \cup \operatorname{Crit}(R \backslash A)$.

Claim 10.4. If $A$ has no cycle, then $|\operatorname{Crit}(A)| \geq|V(A)|-1=|A|$.

In this case $A$ is a tree with $|A|+1$ vertices. We show that at most one non critical vertex may exist in $A$. Suppose on the contrary that $u, v$ are two non-critical vertices in $A$, and let $P=(u, \ldots, v)$ be the (unique) shortest chain between them in $A$. Observe that $P$ has length at least 2 (for otherwise its unique edge $u v$ would satisfy $\operatorname{Crit}(u v)=\emptyset$ ), and that the inclusion into $D^{\prime}$ of the two edges of $P$ incident to $u$ and $v$ does not increase their corresponding indegree or outdegree above $k-1$.

For every white vertex $w$ of $V(P) \backslash\{u, v\}$ select a white head arrow $x w$, and for every black vertex $z$ of $V(P) \backslash\{u, v\}$ select a black tail arrow $z x$ (for twocolored vertices take one such arrow arbitrarily). Let $F$ be the set of selected arrows. So $|F| \leq|P|-1$. Define $R^{*}=(R \backslash P) \cup F$. The graph $D^{*}=D \backslash R^{*}$ belongs to $D(k-1, k-1)$, because the outdegree of every black vertex of $V(P) \backslash$ $\{u, v\}$, and the indegree of every white vertex of $V(P) \backslash\{u, v\}$ is at most $k-1$, furthermore the corresponding degrees of $u$ and $v$ do not increase above $k-1$. The set $R^{*}$ satisfies $\left|R^{*}\right| \leq|R|-1$, contradicting the minimality of $R$. Thus $A$ has at most one non-critical vertex, and Claim 10.4 holds.

Now we consider an arbitrary cycle $C$ in $R$ (if any).

Claim 10.5. $C$ has no edge $e=x y$ with $x \in W \backslash B$ and $y \in B \backslash W$.

Suppose that there is such an edge $e=x y$. Note that $e$ is neither a white head arrow nor a black tail arrow in $C$. Hence $C$ has at most $|C|-1$ white head and black tail arrows. For every white vertex $w \in V(C)$ select a white head arrow $z w$, and for every black vertex $v \in V(C)$ select a black tail arrow $v u$ (for two-colored vertices select one arrow arbitrarily). Let $F$ be the set of $|C|$ selected edges, and define $R^{*}=(R \backslash C) \cup F$. The set $R^{*}$ satisfies $\left|R^{*}\right| \leq|R|$, and contains more white head and black tail arrows than $R$. Furthermore, the graph $D^{*}=D \backslash R^{*}$ belongs to $D(k-1, k-1)$, because the outdegree of every black vertex of $C$, and the indegree of every white vertex of $C$ is at most $k-1$. This contradicts the choice of $R$. Thus Claim 10.5 holds.

Claim 10.6. Let $u, x, y, v$ be four consecutive vertices of $C$.

(1) If $x u, x y, v y \in C$ and $x$ is black, then $y$ is not white.

(2) If $u x, x y, v y \in C$, then either $x$ or $y$ is not white.

(3) If $u x, x y, y v \in C$ and $y$ is black, then $x$ is not white.

Suppose on the contrary that any of (1), (2), (3) fails. Then, in either case, the edge $e=x y$ satisfies $\operatorname{Crit}(e)=\emptyset$, which contradicts the minimality of $R$. Thus Claim 10.6 holds.

Claim 10.7. $C$ is a directed cycle and it is monochromatic, i.e., its vertices are either all in $W \backslash B$ or all in $B \backslash W$. 
Suppose first that $C$ is not a directed cycle, and consider a longest directed subpath $\left(x_{1}, \ldots, x_{q}\right)$ of $C$, where $q \geq 2$.

Suppose that $q=2$, i.e., the directions of the edges alternate on $C$. Let $z_{1} w_{1}, z_{1} w_{2}, z_{2} w_{2} \in C$. If $z_{1} \in W \backslash B$, then by Claim 10.5 we have $w_{1}, w_{2} \in W$. If $z_{1} \in B$, then Claim 10.6 (1) implies that $w_{2} \in B \backslash W$, and $z_{2} \in B$ follows by Claim 10.5. Thus we obtain that either $w_{1}, z_{1}, w_{2} \in W$ or (symmetrically) $z_{1}, w_{2}, z_{2} \in B$. We show a contradiction in the first case, then, by symmetry, the second case is impossible as well. So assume that $w_{1}, z_{1}, w_{2} \in W$ and set $e_{i}=z_{1} w_{i}, i=1,2$. Select an arbitrary white head arrow $f=x z_{1} \in E(D)$. The set $R^{*}=\left(R \backslash\left\{e_{1}, e_{2}\right\}\right) \cup\{f\}$ satisfies $\left|R^{*}\right| \leq|R|-1$, and the graph $D^{*}=D \backslash R^{*}$ belongs to $D(k-1, k-1)$, because $d_{D^{*}}^{-}\left(w_{i}\right)=d_{D^{\prime}}^{-}\left(w_{i}\right) \leq k-1$ for $i=1,2$, and $d_{D^{*}}^{-}\left(z_{1}\right) \leq k-1$. This contradicts the minimality of $R$. Therefore $q \geq 3$.

By Claim 10.6 (2), either $x_{q}$ or $x_{q-1}$ is not white on the directed path $\left(x_{1}, x_{2}, \ldots, x_{q}\right)$, for $q \geq 3$. If $x_{q} \in B \backslash W$, then $x_{q-1} \in B$ by Claim 10.5. Thus that in each case $x_{q-1}$ is black.

Suppose that $q=3$. Let $e_{1}=x_{1} x_{2}, e_{2}=x_{1} y_{2} \in C$, with $e_{1} \neq e_{2}$, and let $y_{3}$ be the second neighbor of $y_{2}$ on $C$ different from $x_{1}$.

Assume first that $y_{2} y_{3} \in E(D)$. Then, by the argument above, $x_{2}$ and $y_{2}$ are both black. Observe that $x_{1} \in W \backslash B$, since otherwise the edges $e_{1}$ and $e_{2}$ have no critical vertices. Now select an arbitrary white head arrow $f=z x_{1} \in E(D)$. The set $R^{*}=\left(R \backslash\left\{e_{1}, e_{2}\right\}\right) \cup\{f\}$ satisfies $\left|R^{*}\right|=|R|-1$, and the graph $D^{*}=D \backslash R^{*}$ belongs to $D(k-1, k-1)$, contradicting the minimality of $R$.

Assume now that $y_{3} y_{2} \in E(D)$ (where $y_{3} y_{2}$ might coincide with $x_{2} x_{3}$, if $C$ is a triangle). As before, we have $x_{2} \in B$ and $x_{1} \in W \backslash B$. Then, by Claim 10.5. $y_{2} \in W$. Selecting a white head arrow $f$ at $x_{1}$ and defining the set $R^{*}=\left(R \backslash\left\{e_{1}, e_{2}\right\}\right) \cup\{f\}$ we obtain a contradiction in the same way as before. Therefore $q \geq 4$.

We already know that $x_{q-1}$ is black. Hence $x_{q-2} \in B \backslash W$ by Claim 10.6 (3). Applying Claim 10.6 (3) repeatedly, we obtain that $x_{q-2}, \ldots, x_{2}$ are in $B \backslash W$. Then we have $x_{1} \in B$ by Claim 10.5. Hence the edge $x_{1} x_{2}$ has no critical vertex, because $d_{D^{\prime}}^{+}\left(x_{1}\right) \leq k-2$ and $d_{D^{\prime}}^{+}\left(x_{2}\right) \leq k-1$, contradicting the minimality of $R$. So we have established that $C$ is a directed cycle.

Now assume without loss of generality that some vertex $y$ of $C$ is black. By Claim 10.6 (3), the predecessor $x \in V(C)$ of $y$ is not white, i.e., it is in $B \backslash W$. Applying Claim 10.6 (3) repeatedly we obtain that every vertex of $C$ is in $B \backslash W$. So $C$ is monochromatic. Thus Claim 10.7 holds.

Claim 10.8. If a component $A$ of $R$ contains a cycle, then $A$ is unicyclic and $|\operatorname{Crit}(A)|=|V(A)|=|A|$.

Let $A$ contain a cycle $C$. By Claim 10.7 and by symmetry, $C$ is a black directed cycle.

For every edge $e=x y \in C$, by Claim 10.3, we have $x \in \operatorname{Crit}(e)$, and by Claim 10.2, we have $d_{R}^{+}(x)=1$. Let $A_{0}$ be a subgraph of $A$ that is maximal with the following property: $A_{0}$ contains $C$, for every $x \in V\left(A_{0}\right)$ there is a directed path in $A_{0}$ from $x$ to some vertex of $C$, and every vertex $x \in V\left(A_{0}\right)$ is 
black and satisfies $d_{R}^{+}(x)=1$. Let us prove that $A_{0}=A$. Note that $A_{0}$ exists, because $C$ itself satisfies all the required properties.

Suppose that $A_{0} \neq A$. Then, since $A$ is connected, there is a vertex $x \in$ $V(A) \backslash V\left(A_{0}\right)$ that is adjacent to some $y \in V\left(A_{0}\right)$. Because $d_{R}^{+}(y)=1$ and $d_{A_{0}}^{+}(y)=1$, we have $e=x y \in A$. Since $y$ is black, Claim 10.3 implies Crit $(e)=$ $\{x\}$. Observe that $A_{0}$ contains a directed $P_{3}$ from $y$ containing black vertices. Hence by Claim 10.6 (3), we have $y \in B \backslash W$. If $x \in W \backslash B$, then let $f=$ $u x \in R$ be any white head arrow. Define $R^{*}=(R \backslash\{e\}) \cup\{f\}$. The digraph $D^{*}=D \backslash R^{*}$ belongs to $D(k-1, k-1)$, because $d_{D^{*}}^{+}(y)=d_{D^{\prime}}^{+}(y) \leq k-1$, and $d_{D^{*}}^{-}(x) \leq k-1$. This contradicts the choice of $R$. So $x$ is black. Because $x$ is black and $x \in \operatorname{Crit}(e)$, Claim 10.2 implies $d_{R}^{+}(x)=1$. Hence one could include $x$ to $A_{0}$, contradicting the maximality of $A_{0}$. Therefore $A_{0}=A$.

Since $A=A_{0}, C$ is the only cycle in $A$ and every vertex $x$ of $A$ is black and satisfies $d_{R}^{+}(x)=1$. Claim 10.3 implies that every vertex of $A$ is a critical vertex of its outgoing edge. So $|\operatorname{Crit}(A)|=|V(A)|=|A|$, and Claim 10.8 holds.

Claims 10.4 and 10.8 show that $|\operatorname{Crit}(A)| \geq|A|$ is true for every connected component $A$. If $A_{1}, \ldots, A_{t}$ are the components of $R$, we have clearly $\operatorname{Crit}(R)=\operatorname{Crit}\left(A_{1}\right) \cup \cdots \cup \operatorname{Crit}\left(A_{t}\right)$. Thus we obtain $|\operatorname{Crit}(R)| \geq|R|$, which proves Claim 10.1. This concludes the proof of the theorem.

The regular tournament on $2 k+1$ vertices has indegree equal to outdegree for every vertex, hence it is in $D(k, k)$. To obtain a subgraph belonging to $D(k-1, k-1)$ one has to remove at least $k+1$ from its $m=\left(\begin{array}{c}2 k+1 \\ 2\end{array}\right)$ edges. This shows that the tournament has no subgraph in $D(k-1, k-1)$ containing more than $\left(\begin{array}{c}2 k+1 \\ 2\end{array}\right)-k+1=m-(1+1 / k) m /(2 k+1)=(2 k-1 / k) m /(2 k+1)$ edges.

Corollary 11. Every digraph $D \in D(2,2)$ with $m$ edges contains a subgraph belonging to $D(1,1)$ with at least $3 \mathrm{~m} / 5$ edges.

We note that for $k=1$, Theorem 10 yields another proof that every digraph $D \in D(1,1)$ with $m$ edges contains a directed cut of size at least $m / 3$ (cf. Corollary [3).

\section{Cuts in $D(k, k)$}

In [1] it was observed that the $k$-regular orientation of the complete graph on $2 k+1$ vertices has no directed cut of size more than $\left(\frac{1}{4}+\frac{1}{8 k+4}\right)\left(\begin{array}{c}2 k+1 \\ 2\end{array}\right)$. Consequently, in a digraph $D \in D(k, k)$ with $m$ edges one cannot guarantee a directed cut of size larger than $\left(\frac{1}{4}+\frac{1}{8 k+4}\right) m$. It was proved in [1] that every digraph with outdegree at most $k$ does contain a directed cut of that size. Using the same methods we show that it is also true for the acyclic members of $D(k, k)$.

The basic tool is a lemma in [10, that is proved there by elementary counting.

Lemma 1 (10). If a $\gamma$-colorable graph $G$ has $m$ edges, then it has a bipartite partial graph with at least $\left(\left\lfloor\gamma^{2} / 4\right\rfloor /\left(\begin{array}{c}\gamma \\ 2\end{array}\right)\right) m$ edges. 
Theorem 12. If $D \in D(k, k)$ is acyclic and has $m$ edges, then $D$ contains a directed cut of size at least $\left(\frac{1}{4}+\frac{1}{8 k+4}\right) m$.

Proof. Let $D^{+}$be the subgraph of $D$ induced by the set $X=\left\{v \in V(D) \mid d^{+}(v) \leq\right.$ $k\}$ and let $D^{-}$be the subgraph of $D$ induced by $V(D) \backslash X$. Because $D$ is acyclic, every subgraph of $D^{+}$has a source, thus its underlying graph $G^{+}$is $k$-degenerate. Similarly, every subgraph of $D^{-}$has a sink, thus its underlying graph $G^{-}$is $k$-degenerate. Consequently, both graphs $G^{+}$and $G^{-}$are $(k+1)$ colorable, therefore the underlying graph $G$ of $D$ is $(2 k+2)$-colorable.

Applying Lemma 1 with $\gamma=2 k+2$, we obtain a bipartite partial graph of $G$ with $\frac{k+1}{2 k+1} m$ edges. In $D$ at least half of the edges of that bipartite graph form a directed cut of size at least $\frac{k+1}{4 k+2} m=\left(\frac{1}{4}+\frac{1}{8 k+4}\right) m$.

We do not know whether Theorem 12 remains true for all digraphs in $D(k, k)$, and for every $k$. The coefficients are $1 / 3,3 / 10$ and $2 / 7$ for $k=1,2$, and 3 , respectively. By Theorem 7 a digraph $D \in D(1,1)$ of size $m$ has a cut with at least $m / 3$ edges. The theorem below answers the question affirmatively for $D(2,2)$.

Theorem 13. Every digraph $D \in D(2,2)$ with $m$ edges has a directed cut of size at least $3 \mathrm{~m} / 10$.

Proof. We prove the theorem by induction on $m$. For $m=1$ the theorem is true. Now suppose that $m \geq 2$ and that the theorem holds for every digraph with at most $m-1$ edges. Since $D$ is in $D(2,2)$, its vertex set can be partitioned into two sets $X, Y$ such that every vertex $x \in X$ satisfies $d^{-}(x) \leq 2$ and every vertex $y \in Y$ satisfies $d^{+}(y) \leq 2$. Consider the set of edges $F=\{x y \in E \mid x \in X, y \in Y\}$.

First suppose that the underlying bipartite graph $(X, Y ; F)$ contains a cycle. Let $C$ be any such cycle, say with length $2 k$, let $X_{C}$ and $Y_{C}$ be the set of vertices of $C$ that lie in $X$ and $Y$ respectively, and let $F_{C}$ be the set of edges of $C$. So $\left|F_{C}\right|=2 k$. Let $E_{C}$ be the set of edges such that either their end is in $X_{C}$ or their origin is in $Y_{C}$. By the definition of $X, Y$ and the fact that $D$ is in $D(2,2)$, we have $\left|E_{C}\right| \leq 4 k$. Consider the digraph $D^{\prime}=D \backslash\left(E_{C} \cup F_{C}\right)$. Clearly, $D^{\prime} \in D(2,2)$, and the number $m^{\prime}$ of edges of $D^{\prime}$ satisfies $m^{\prime} \geq m-6 k$. By the induction hypothesis, $D^{\prime}$ has a directed cut of size at least $3 \mathrm{~m}^{\prime} / 10$. If the edges of $F_{C}$ are added to such a directed cut, we obtain a directed cut of $D$, because $D^{\prime}$ does not contain any edge of $E_{C}$. This directed cut of $D$ has size at least $3 m^{\prime} / 10+\left|F_{C}\right| \geq 3(m-6 k) / 10+2 k \geq 3 m / 10$. So the theorem holds for $D$.

Now suppose that the bipartite graph $(X, Y ; F)$ does not contain any cycle. Thus $|F| \leq n-1$, where $n$ is the number of vertices of $D$. By the definition of $X$ and $Y$, we have $m \leq 2|X|+2|Y|+|F| \leq 2 n+n-1=3 n-1$, which implies that $D$ has a vertex $v$ of degree at most 5 . Actually the same argument can be repeated with $D \backslash\{v\}$, and so on. Thus the underlying graph of $D$ is 5 -degenerate and therefore has chromatic number at most 6 . Applying Lemma 1 with $\gamma=6$ we obtain a bipartite subgraph with $3 / 5$ edges, thus $D$ has a directed cut of size at least $3 m / 10$. 


\section{Problems}

Let $c_{\max }$ be the ratio of the maximum directed cut size to the edge count $m$ of a digraph. For connected digraphs of $D(1,1)$, Theorem 5 improves the basic estimation $c_{\max } \geq 1 / 3$ to $c_{\max } \geq 7 / 20$ provided $m>3$. On the other hand, Example 1 before Theorem 1 shows infinitely many connected digraphs of $D(1,1)$ with $c_{\max }<3 / 8$. We conjecture that the bound $c_{\max } \geq 7 / 20$ can be improved to $3 / 8$ in the limit in the following sense.

Problem 1. For every $\varepsilon>0$, there exists a constant $m_{\varepsilon}$ such that $c_{\max }>$ $3 / 8-\varepsilon$ holds for every connected digraph of $D(1,1)$ with $m>m_{\varepsilon}$ edges.

At some point of the investigation in $D(1,1)$ we observed that the presence of source or sink vertices of the digraph increases the size of a maximum directed cut. Corollary 4 might have the following sharpening.

Problem 2. If a connected digraph $D \in D(1,1)$ with $m$ edges contains no directed triangle and has $s$ vertices with indegree or outdegree zero, then $D$ has a directed cut of size at least $(2 m+s) / 5$.

Bondy and Locke [4] proved that a triangle-free subcubic graph has a cut (a bipartite subgraph) of size at least $4 \mathrm{~m} / 5$. A characterization of all extremal graphs for that bound was given by $\mathrm{Xu}$ and $\mathrm{Yu}$ in [11. The problem of characterizing the extremal graphs for the bound of Corollary 4 remains open:

Problem 3. Determine the list of all digraphs $D \in D(1,1)$ of size $m$ that contain no directed triangle and have no directed cut with more than $2 \mathrm{~m} / 5$ edges.

Bondy and Locke's result in [4] consists of a polynomial time algorithm that finds a cut with at least $4 \mathrm{~m} / 5$ edges in a triangle-free subcubic graph. It is known that finding a maximum cut is NP-hard even in the restricted family of triangle-free cubic graphs (see Yannakakis [12]). Even the approximation of the max cut problem in cubic graphs within the ratio of 0.997 is NP-hard (see Berman and Karpinski [3]). On the other hand, Halperin, Livnat and Zwick [9] give a polynomial time approximation algorithm with ratio 0.9326 .

Concerning digraphs in $D(1,1)$, Corollary 6 gives a polynomial time algorithm that produces a cut of size at least $7 \mathrm{~m} / 20$ in every digraph in $D(1,1)$ of which no component is a directed triangle; and so this is an approximation algorithm with ratio 0.35. Can a better ratio be obtained? Actually, as far as we know, none of the known results implies that computing the exact value of a maximum directed cut is NP-hard in $D(1,1)$. So we ask:

Problem 4. What is the complexity status of computing the size of a maximum directed cut in a digraph of $D(1,1)$ ? If it is NP-hard, what is the best value of $\varepsilon$ for which there is a polynomial time approximation algorithm with ratio $1-\varepsilon$ for this problem? 
The same problem can be posed for digraphs of $D(1,1)$ with no directed triangle, or with no triangle at all.

How large a subgraph belonging to $D(1,1)$ can be found in a digraph $D \in$ $D(2,2)$ ? Corollary 11 says that $D$ with $m$ edges contains a subgraph in $D(1,1)$ with at least $3 \mathrm{~m} / 5$ edges. This lower bound is probably not sharp.

Problem 5. Determine the largest constant $\lambda$ such that in every digraph $D \in$ $D(2,2)$ with $m$ edges there exists a subgraph $D^{\prime} \in D(1,1)$ of size at least $\lambda m$.

If $D$ is the regular tournament on five vertices, then $D \in D(2,2)$ and one needs to remove at least three edges to obtain a subgraph $D^{\prime} \in D(1,1)$. This shows that in the problem above $\lambda \leq 7 / 10$.

From a result in 1 it follows that the edges of every graph $D \in D(2,2)$ can be decomposed into at most five directed cuts. Furthermore, four cuts are sufficient if $D$ is acyclic. The regular tournament on five vertices shows that four cuts might be necessary. Indeed, it has 10 edges, and the size of a directed cut is at most 3. No example has been found to show that five directed cuts are necessary.

Problem 6. The edges of every digraph $D \in D(2,2)$ can be decomposed into at most four directed cuts.

Several problems remain open in $D(2,2)$ pertaining to the ratio $c_{\max }$.

Problem 7. If $D \in D(2,2)$ has $m$ edges and contains no copy of the regular tournament on five vertices, then $D$ has a directed cut of size at least $m / 3$.

We do not know whether Theorem 12 pertaining to acyclic digraphs remains true for all digraphs in $D(k, k)$, and for every $k$. The coefficients $\frac{1}{4}+\frac{1}{8 k+4}$ are equal to $1 / 3,3 / 10$, and $2 / 7$ for $k=1,2$, and 3 , respectively. By Corollary 3 , and by Theorem 13, a digraph $D$ of size $m$ has a cut with $m / 3$ and $3 m / 10$ edges, respectively for $D \in D(1,1)$ and $D \in D(2,2)$. The next case $k=3$ is proposed here as a question. It is quite possible that the answer is negative. Even if it is not the case we conjecture that Theorem 12 does not extend for every $k$.

Problem 8. Is it true that every digraph of $D(3,3)$ with $m$ edges contains a directed cut of size at least $2 \mathrm{~m} / 7$ ?

Digraphs with maximum outdegree $k$ satisfy $c_{\max } \geq \frac{1}{4}+\frac{1}{8 k+4}$, and this is the best bound, as shown in [1]. It is worth noting that the same bound was obtained here in Theorem 12 for acyclic members of $D(k, k)$. Furthermore, the regular tournament on $2 k+1$ vertices is an example of a digraph with no directed cut larger than $\left(\frac{1}{4}+\frac{1}{8 k+4}\right) m$. We believe that in the larger family $D(k, k)$ there are more examples showing that this bound cannot be achieved, provided $k$ is large enough.

Problem 9. There exists a $k_{0}$ such that for every $k \geq k_{0}$ there are digraphs in $D(k, k)$ with $c_{\max }<\frac{1}{4}+\frac{1}{8 k+4}$. 
In Theorem 10 we are dealing with the largest subgraph of $D \in D(k, k)$ that belongs to the "lower" class $D(k-1, k-1)$. This leads naturally to the investigation of the minimum sets $R \subset E(D)$ to be removed from $D$ in order to lower its class. The proof of Theorem 10 suggests that such minimum sets considered as digraphs have a particular structure reminiscent of forests. Repeating the procedure, one obtains a decomposition of the original digraph $D \in D(k, k)$ into at most $k$ of these structures.

Practical applications motivate the study of decompositions of digraphs into directed stars (see [5]). The directed star arboricity (dst) introduced in [8] is defined as the minimum number of outstar forests (also called galaxy) the edge set of a digraph can be partitioned. For instance it is proved in [2 that a digraph $D$ with indegree at most $k$ has a decomposition into $k$ outforests plus one galaxy. This result implies $d s t(D) \leq 2 k+1$, and it is conjectured in [2] that $2 k$ is the tight bound, for $k \geq 2$.

As a general problem we propose here a similar decomposition theory of the digraphs of $D(k, k)$ into appropriate forest-like structures.

\section{Acknowledgment}

The first author's participation in the present research was supported by the Professional Development Assignment obtained from The University of Memphis and by the funding of the Universite de Grenoble 1, Joseph Fourier. He

is grateful for the help and the hospitality of all colleagues at the Laboratoire Leibniz - IMAG during his stay in Grenoble in October and November 2006.

This research has been supported by the ADONET network of the European Community, which is a Marie Curie Training Network.

\section{References}

[1] N. Alon, A. Gyárfás, B. Bollobás, J. Lehel and A. Scott, Maximum directed cuts in acyclic digraphs, J. Graph Theory 55 (2007), 1-13.

[2] O. Amini, F. Havet, F. Huc, and S. Thomassé, Directed star arboricity of digraphs, Journées Graphes et Algorithmes, Orléans, Novembre 2006.

[3] P. Berman, M. Karpinski, On some tighter inapproximability results (extended abstract) Lecture Notes in Comput. Sci., 1644, Springer, Berlin (1999), 200-209.

[4] J. A. Bondy and S.C. Locke, Largest bipartite subgraphs in triangle-free graphs with maximum degree three, J. Graph Theory 10 (1986), 477-504.

[5] R. Brandt, and T.F. Gonzalez, Wavelength assignment in multifiber optical star networks under the multicasting communication mode, Journal of Interconnection Networks 6 (2005), 383-405. 
[6] M. Cropper, M. Jacobson, A. Gyárfás, and J. Lehel, The Hall-ratio of graphs and hypergraphs, Les cahiers du Laboratoire Leibniz, No 17, Dec. 2000 .

[7] R. Diestel. Graph Theory. Graduate Texts in Mathematics 173, Springer, 1997.

[8] B. Guiduli, On incidence coloring and star arboricity of graphs, Discrete Math. 163 (1997), 275-278.

[9] E. Halperin, D. Livnat, U. Zwick. Max Cut in cubic graphs. J. Algorithms 53 (2004), 169-185.

[10] J. Lehel and Zs. Tuza, Triangle-free partial graphs and edge covering theorems, Discrete Math. 39 (1982), 59-65.

[11] B. Xu and X. Yu, Triangle-free subcubic graphs with minimum bipartite density, manuscript.

[12] M. Yannakakis, Node- and edge-deletion NP-complete problems. Conference Record of the Tenth Annual ACM Symposium on Theory of Computing (San Diego, Calif., 1978), ACM, New York (1978), pp. 253-264. 